\title{
ERRATUM
}

Genji Saito · Kozo Uto • Kohei Kazahaya •

Hiroshi Shinohara • Yoshihisa Kawanabe ·

Hisao Satoh

\section{Petrological characteristics and volatile content of magma from the 2000 eruption of Miyakejima Volcano, Japan}

Published online: 14 June 2005

(C) Springer-Verlag 2005

\section{Bull Volcanol (2005) 67(3): 268-280}

In the original online publication and the print version of this article, Tables 1 and 2 were unfortunately omitted. We apologise for this error. The tables are printed below.

The online version of the original article can be found at http://dx.doi.org/10.1007/s00445-004-0409-z.

G. Saito $(\bowtie) \cdot$ K. Uto · K. Kazahaya · H. Shinohara ·

Y. Kawanabe

Geological Survey of Japan, National Institute of Advanced

Industrial Science and Technology,

Central 7, Higashi 1-1-1,

Tsukuba, Ibaraki 305-8567, Japan

e-mail: saito-g@aist.go.jp

Tel.: +81-29-861-3893

Fax: +81-29-856-8725

H. Satoh

Faculty of Science, Tohoku University,

Aoba-ku,

Sendai 980-8577, Japan 
Table 1 Major element composition of eruptives on 18 August 2000 of Miyakejima volcano

\begin{tabular}{lllllllll}
\hline Sample No. & Bomb 819B1 & Lapilli 1-a & Lapilli 2-b & Lapilli 3-c & Lapilli 3-d & Lapilli 3-f & Lapilli 4-b & Black ash ${ }^{2}$ 4-5 \\
\hline $\mathrm{SiO}_{2}$ & 50.63 & 50.70 & 50.67 & 50.49 & 50.77 & 50.63 & 50.90 & 50.36 \\
$\mathrm{TiO}_{2}$ & 1.12 & 1.11 & 1.12 & 1.12 & 1.16 & 1.12 & 1.13 & 1.17 \\
$\mathrm{Al}_{2} \mathrm{O}_{3}$ & 17.73 & 17.84 & 17.64 & 17.73 & 17.65 & 18.02 & 17.82 & 16.77 \\
$\mathrm{FeO}^{*}$ & 11.14 & 11.06 & 11.14 & 11.18 & 11.29 & 11.21 & 11.23 & 11.34 \\
$\mathrm{MnO}$ & 0.17 & 0.17 & 0.17 & 0.17 & 0.18 & 0.17 & 0.17 & 0.18 \\
$\mathrm{MgO}$ & 4.01 & 3.99 & 4.02 & 4.00 & 4.15 & 4.17 & 4.05 & 4.18 \\
$\mathrm{CaO}$ & 10.97 & 11.03 & 10.94 & 10.93 & 10.94 & 10.95 & 10.96 & 10.54 \\
$\mathrm{Na}_{2} \mathrm{O}$ & 2.34 & 2.40 & 2.40 & 2.30 & 2.37 & 2.38 & 2.42 & 2.31 \\
$\mathrm{~K}_{2} \mathrm{O}$ & 0.40 & 0.40 & 0.40 & 0.40 & 0.41 & 0.39 & 0.40 & 0.42 \\
$\mathrm{P}_{2} \mathrm{O}_{5}$ & 0.11 & 0.12 & 0.12 & 0.12 & 0.13 & 0.11 & 0.12 & 0.12 \\
$\mathrm{Total}$ & 98.62 & 98.82 & 98.62 & 98.44 & 99.05 & 99.15 & 99.20 & 97.39 \\
\hline
\end{tabular}

$\mathrm{FeO}^{*}$, total iron as $\mathrm{FeO}$.

A Bomb (819B1) was collected as Miyake-Village stock farm (see Fig. 1). Sampling points for lapilli and ash are as follows; 1-a and 2-b at an observation point $\mathrm{C}$ in Fig. 1, 3-c, 3-d and 3-f at a point D, and 4-b and 4-5 at a point F (see Fig. 1).

Analytical errors for each element were shown in Saito et al. (2002).

${ }^{1}$ Out side part of a cored bomb, which contains a fragment of old lava inside.

${ }^{2}$ Black ash particles (1-2 mm) having similar texture to the bomb (819B1).

Table 2 Descriptions and chemical analyses of representative melt inclusions in lapilli and groundmass of a bomb by the 18 August eruption of Miyakejima volcano

\begin{tabular}{|c|c|c|c|c|c|c|c|c|c|c|}
\hline \multirow{2}{*}{$\begin{array}{l}\text { Sample } \\
\text { Inclusion No. }{ }^{\mathrm{a}}\end{array}$} & \multicolumn{10}{|c|}{ Melt inclusions in plagioclase } \\
\hline & 1-a-1-1 & 1-a-2-1 & 1-a-3-1 & 1-b-1-1 & 1-gs-4-1 & 1-gs-1-1 & 2-b-2-1 & 2-b-3-1 & 2-b-4-1 & $2-b-4-2$ \\
\hline Host chemistry ${ }^{b}$ & An87 & An88 & An87 & An89 & An88 & An88 & An88 & An87 & An84 & An88 \\
\hline Inclusion size $(\mu \mathrm{m})$ & $150 \times 60$ & $80 \times 50$ & $80 \times 50$ & $80 \times 60$ & $130 \times 30$ & $>30 \times 40$ & $160 \times 90$ & $160 \times 50$ & $70 \times 50$ & $80 \times 50$ \\
\hline Bubble in inclusion $^{c}$ & & $5 b(4)$ & $1 b(2)$ & $2 b(15)$ & $2 b(15)$ & & $4 b(23)$ & $5 b(23)$ & $1 b(18)$ & $3 b(11)$ \\
\hline Bubble $(\operatorname{vol} \%)^{\mathrm{d}}$ & 0 & 0.03 & 0.004 & 2 & 3 & 0 & 1 & 5 & 2 & 0.9 \\
\hline Overgrowth $(\text { vol\%) })^{\mathrm{e}}$ & 2 & 10 & 10 & 9 & 14 & & 3 & 11 & 16 & 6 \\
\hline $\mathrm{SiO}_{2}$ & 51.44 & 50.29 & 51.52 & 51.21 & 52.54 & n.a. & 50.26 & 51.94 & 51.67 & 51.32 \\
\hline $\mathrm{TiO}_{2}$ & 1.36 & 1.40 & 1.26 & 1.26 & 1.31 & n.a. & 1.27 & 1.39 & 1.44 & 1.32 \\
\hline $\mathrm{Al}_{2} \mathrm{O}_{3}$ & 13.26 & 12.92 & 12.64 & 12.84 & 12.06 & n.a. & 13.44 & 12.67 & 11.31 & 12.61 \\
\hline $\mathrm{FeO}^{\mathrm{f}}$ & 12.93 & 13.80 & 13.60 & 13.37 & 13.21 & n.a. & 13.56 & 14.39 & 13.13 & 12.83 \\
\hline $\mathrm{MnO}$ & 0.29 & 0.13 & 0.17 & 0.22 & 0.25 & n.a. & 0.26 & 0.31 & 0.24 & 0.23 \\
\hline $\mathrm{MgO}$ & 4.67 & 5.16 & 5.33 & 5.10 & 5.43 & n.a. & 5.04 & 5.07 & 5.32 & 5.25 \\
\hline $\mathrm{CaO}$ & 8.02 & 7.94 & 8.13 & 8.81 & 8.51 & n.a. & 8.90 & 8.78 & 8.14 & 8.68 \\
\hline $\mathrm{Na}_{2} \mathrm{O}$ & 2.95 & 2.32 & 2.85 & 2.23 & 2.39 & n.a. & 2.11 & 2.62 & 2.19 & 2.30 \\
\hline $\mathrm{K}_{2} \mathrm{O}$ & 0.54 & 0.65 & 0.63 & 0.64 & 0.70 & n.a. & 0.52 & 0.71 & 0.71 & 0.53 \\
\hline $\mathrm{P}_{2} \mathrm{O}_{5}$ & 0.16 & 0.14 & 0.15 & 0.11 & 0.10 & n.a. & 0.07 & 0.12 & 0.07 & 0.09 \\
\hline $\mathrm{H}_{2} \mathrm{O}^{\mathrm{g}}$ & 1.25 & 1.21 & 1.32 & 1.22 & 1.22 & 1.55 & 1.33 & 1.01 & 0.90 & 1.37 \\
\hline $\mathrm{CO}_{2}(\mathrm{ppm})^{\mathrm{h}}$ & $<41$ & $38 / 42$ & $50 / 51$ & $74 / 630$ & $<66 /<660$ & $<94$ & $<47 /<200$ & $<31 /<500$ & $<47 /<300$ & $<53 /<200$ \\
\hline $\mathrm{S}(\mathrm{ppm})$ & 570 & 860 & 1190 & 1180 & 900 & n.a. & 1230 & 910 & 850 & 1050 \\
\hline $\mathrm{Cl}(\mathrm{ppm})$ & 690 & 640 & 630 & 640 & 630 & n.a. & 680 & 680 & 620 & 670 \\
\hline Total & 97.01 & 96.11 & 97.76 & 97.19 & 97.86 & & 96.94 & 99.14 & 95.26 & 96.70 \\
\hline Sat. Press. $(\mathrm{MPa})^{\mathrm{i}}$ & 24 & $23 / 24$ & $28 / 28$ & $31 / 150$ & $<29 /<150$ & $<45$ & $<28 /<62$ & $<17 /<115$ & $<18 /<73$ & $<30 /<63$ \\
\hline \multicolumn{11}{|l|}{ Sample } \\
\hline Inclusion No. ${ }^{\mathrm{a}}$ & $2-b-4-3$ & $2-b-5-1$ & 2-gs-2-1 & 2-gs-3-1 & 2-gs-3-2 & 2-gs-4-1 & 2-gs-6-1 & 3-a-2-1 & 3-a-2-2 & 4-gs-3-1 \\
\hline Host chemistry ${ }^{b}$ & An85 & An87 & An89 & & An87 & An84 & An84 & An89 & An88 & An88 \\
\hline Inclusion size $(\mu \mathrm{m})$ & $80 \times 70$ & $90 \times 70$ & $40 \times 30$ & $40 \times 30$ & $40 \times 30$ & $120 \times 60$ & $160 \times 60$ & $160 \times 110$ & $70 \times 50$ & $120 \times 60$ \\
\hline Bubble in inclusion $^{c}$ & $1 b(25)$ & $2 b(7)$ & $1 b(18)$ & $1 b(14)$ & $1 b(14)$ & $1 b(28)$ & $1 b(19)$ & $1 b(27)$ & $1 b(15)$ & $4 b(22)$ \\
\hline Bubble $\left(\right.$ vol\%) ${ }^{\mathrm{d}}$ & 2 & 0.1 & 13 & 9 & 8 & 6 & 1 & 1 & 2 & 3 \\
\hline Overgrowth $(\text { vol\%) })^{\mathrm{e}}$ & 8 & 16 & 10 & & 24 & & 4 & 7 & 12 & 5 \\
\hline $\mathrm{SiO}_{2}$ & 52.73 & 50.56 & 53.69 & n.a. & 51.55 & n.a. & 51.97 & 52.14 & 51.88 & 52.39 \\
\hline $\mathrm{TiO}_{2}$ & 1.34 & 1.75 & 1.46 & n.a. & 1.19 & n.a. & 1.12 & 1.24 & 1.57 & 1.33 \\
\hline $\mathrm{Al}_{2} \mathrm{O}_{3}$ & 12.68 & 9.13 & 11.77 & n.a. & 8.41 & n.a. & 13.62 & 13.60 & 12.94 & 13.54 \\
\hline $\mathrm{FeO}^{1}$ & 12.91 & 16.32 & 14.57 & n.a. & 18.95 & n.a. & 13.04 & 13.30 & 12.73 & 13.38 \\
\hline $\mathrm{MnO}$ & 0.26 & 0.34 & 0.23 & n.a. & 0.31 & n.a. & 0.21 & 0.26 & 0.22 & 0.23 \\
\hline $\mathrm{MgO}$ & 5.05 & 6.39 & 5.24 & n.a. & 6.80 & n.a. & 4.88 & 4.93 & 5.14 & 5.24 \\
\hline $\mathrm{CaO}$ & 8.74 & 8.91 & 8.80 & n.a. & 7.97 & n.a. & 8.84 & 8.78 & 8.68 & 9.05 \\
\hline
\end{tabular}


Table 2 Continued.

\begin{tabular}{|c|c|c|c|c|c|c|c|c|c|c|}
\hline $\begin{array}{l}\text { Sample } \\
\text { Inclusion No. }^{\mathrm{a}}\end{array}$ & $2-b-4-3$ & 2-b-5-1 & 2-gs-2-1 & 2-gs-3-1 & 2-gs-3-2 & 2-gs-4-1 & 2-gs-6-1 & $3-a-2-1$ & 3-a-2-2 & 4-gs-3-1 \\
\hline $\mathrm{Na}_{2} \mathrm{O}$ & 2.79 & 1.54 & 1.96 & n.a. & 1.63 & n.a. & 2.49 & 2.35 & 1.77 & 2.38 \\
\hline $\mathrm{K}_{2} \mathrm{O}$ & 0.62 & 0.55 & 0.63 & n.a. & 0.69 & n.a. & 0.60 & 0.66 & 0.70 & 0.59 \\
\hline $\mathrm{P}_{2} \mathrm{O}_{5}$ & 0.08 & 0.08 & 0.17 & n.a. & 0.14 & n.a. & 0.16 & 0.08 & 0.16 & 0.23 \\
\hline $\mathrm{H}_{2} \mathrm{O}^{\mathrm{g}}$ & 1.60 & 1.27 & $>0.6$ & $>0.9$ & $>0.6$ & $>1.2$ & 1.43 & 1.53 & 1.66 & 1.92 \\
\hline $\mathrm{CO}_{2}(\mathrm{ppm})^{\mathrm{h}}$ & $<53 /<440$ & $<45 /<64$ & n.a. & n.a. & n.a. & n.a. & $<70 /<400$ & $<58 /<280$ & $<56 /<390$ & $<100 /<200$ \\
\hline $\mathrm{S}(\mathrm{ppm})$ & 900 & 1110 & 1400 & n.a. & 1510 & n.a. & 970 & 1030 & 880 & 1090 \\
\hline $\mathrm{Cl}(\mathrm{ppm})$ & 650 & 670 & 770 & n.a. & 770 & n.a. & 740 & 670 & 620 & 660 \\
\hline Total & 98.94 & 97.02 & 98.73 & & 97.86 & & 98.52 & 99.03 & 97.60 & 100.45 \\
\hline $\begin{array}{l}\text { Sat. Press. }(\mathrm{MPa})^{\mathrm{i}} \\
\text { Sample }\end{array}$ & \multicolumn{6}{|c|}{$\begin{array}{l}<37 /<120 \quad<26 /<30 \\
\text { Melt inclusions in olivine }\end{array}$} & $\begin{array}{l}<36 /<110 \\
\quad \text { Melt inclu }\end{array}$ & $\begin{array}{l}<36 /<83 \\
\text { usions in cpx }\end{array}$ & \multicolumn{2}{|c|}{$\begin{array}{l}<40 /<110 \quad 60 / 280 \\
\text { Groundmass of a bomb }\end{array}$} \\
\hline Inclusion No. ${ }^{\mathrm{a}}$ & 1-gs-2-1 ${ }^{\mathrm{m}}$ & \multicolumn{2}{|l|}{ 2-gs-5-1 } & $3-c-1-1$ & $3-c-2-1$ & 4-gs-2-1 & \multicolumn{2}{|c|}{ 1-a-4-1 $\mathbf{1}^{\mathrm{j}}$} & \multicolumn{2}{|c|}{ Groundmass of a bomb } \\
\hline Host chemistry ${ }^{b}$ & Fo73 & Fo71 & & Fo76 & Fo71 & Fo71 & \multicolumn{2}{|c|}{ Wo40En43(Mg\#73) } & & \\
\hline Inclusion size $(\mu \mathrm{m})$ & $50 \times 50$ & \multirow[t]{2}{*}{$60 \times 40$} & \multicolumn{2}{|c|}{$60 \times 60$} & $30 \times 20$ & $70 \times 50$ & \multicolumn{2}{|l|}{$10 \times 10$} & & \\
\hline Bubble in inclusion ${ }^{\mathrm{c}}$ & & & \multirow{2}{*}{\multicolumn{2}{|c|}{0}} & & $1 b(12)$ & & & & \\
\hline Bubble $(\operatorname{vol} \%)^{\mathrm{d}}$ & 0 & 0 & & & 0 & 1 & \multicolumn{2}{|l|}{0} & & \\
\hline Overgrowth $(\text { vol\%) })^{\mathrm{e}}$ & 8 & 2 & \multicolumn{2}{|c|}{$10^{1}$} & 5 & 3 & \multicolumn{2}{|l|}{21} & \multirow{2}{*}{\multicolumn{2}{|c|}{$53.39(0.90)$}} \\
\hline $\mathrm{SiO}_{2}$ & 50.75 & 55.89 & \multicolumn{2}{|c|}{50.23} & 53.23 & 54.16 & \multicolumn{2}{|l|}{57.57} & & \\
\hline $\mathrm{TiO}_{2}$ & 1.40 & 1.37 & \multicolumn{2}{|c|}{1.33} & 1.42 & 1.40 & \multicolumn{2}{|l|}{1.23} & \multicolumn{2}{|l|}{$1.31(0.14)$} \\
\hline $\mathrm{Al}_{2} \mathrm{O}_{3}$ & 14.19 & 15.20 & \multicolumn{2}{|c|}{18.20} & 16.09 & 15.13 & 17.56 & & $14.99(1.05$ & \\
\hline $\mathrm{FeO}^{\mathrm{f}}$ & 10.27 & 11.43 & & 0.33 & 10.47 & 11.48 & 8.97 & & $12.32(0.68$ & \\
\hline $\mathrm{MnO}$ & 0.12 & 0.22 & & .23 & 0.19 & 0.24 & 0.24 & & $0.22(0.08)$ & \\
\hline $\mathrm{MgO}$ & 3.38 & 3.07 & & .06 & 2.75 & 3.54 & 1.60 & & $5.03(0.36)$ & \\
\hline $\mathrm{CaO}$ & 8.88 & 10.34 & & 2.46 & 10.79 & 9.68 & 6.53 & & $9.44(0.51)$ & \\
\hline $\mathrm{Na}_{2} \mathrm{O}$ & 2.93 & 2.40 & & 2.71 & 2.36 & 2.66 & 3.22 & & $2.61(0.60)$ & \\
\hline $\mathrm{K}_{2} \mathrm{O}$ & 0.72 & 0.50 & & .33 & 0.58 & 0.59 & 0.77 & & $0.58(0.11)$ & \\
\hline $\mathrm{P}_{2} \mathrm{O}_{5}$ & 0.12 & 0.24 & & .07 & 0.09 & 0.21 & 0.24 & & $0.10(0.08)$ & \\
\hline $\mathrm{H}_{2} \mathrm{O}^{\mathrm{g}}$ & $>0.9$ & 1.39 & & $>$ n.a. & $>$ n.a. & $>1.37$ & $>$ n.a. & & & \\
\hline $\mathrm{CO}_{2}(\mathrm{ppm})^{\mathrm{h}}$ & n.a. & $<73$ & & l.a. & n.a. & $<84 /<360$ & n.a. & & & \\
\hline $\mathrm{S}(\mathrm{ppm})$ & 790 & 660 & & 710 & 1060 & 650 & 640 & & & \\
\hline $\mathrm{Cl}(\mathrm{ppm})$ & 730 & 950 & & 580 & 720 & 830 & 840 & & & \\
\hline Total & 92.91 & 102.19 & & 9.18 & 98.14 & 100.60 & 98.07 & & 100.00 & \\
\hline Sat. Press. $(\mathrm{MPa})^{\mathrm{i}}$ & & $<35$ & & & & $<37 /<96$ & & & & \\
\hline
\end{tabular}

In weight percent except for $\mathrm{CO}_{2}$, sulfur and chlorine concentrations; n.a. = not analysed.

${ }^{a}$ First numbers represent sampling locations. Sampling locations are as follows; 1 and 2 at an observation point $\mathrm{C}$ in Fig. 1, 3 at a point D and 4 at a point F. Second alphabet is for identification of each lapilli. Third and fourth number of each sample are to distinguish each phenocryst and each inclusion.

${ }^{\mathrm{b}}$ Chemical composition of host phenocrysts near the melt inclusions. An = anorthite content $(\mathrm{mol} \%)$ in the plagioclase, En $=$ enstatite content $(\mathrm{mol} \%)$ in pyroxene, $\mathrm{W}=$ wollastonite content $(\mathrm{mol} \%)$ in clinopyroxene.

${ }^{c}$ Numbers with $b$ represent the number of bubbles in each melt inclusion. Numbers in parentheses represent size of bubbles $(\mu \mathrm{m})$.

${ }^{\mathrm{d}}$ Volume percent of bubble in inclusion. See text.

${ }^{\text {e}}$ Extent of overgrowth in inclusion. See text.

${ }^{\mathrm{f}}$ All iron calculated as FeO.

${ }^{g}$ Values of volatile concentrations $\left(\mathrm{H}_{2} \mathrm{O}, \mathrm{CO}_{2}, \mathrm{~S}\right.$ and $\left.\mathrm{Cl}\right)$ were corrected for the overgrowth (see text). The correction was as follows; if the overgrowth was $\times$ vol\%, the analytical value was multiplied by $(1-0.01 \times)$ to be the corrected value. Water concentrations of Nos. 2-gs-2-1, 2-gs-3-1, 2-gs-3-2, 2-gs-4-1 and 1-gs-2-1 are minimum estimates because these samples were single side polished.

${ }^{\mathrm{h}} \mathrm{x} / \mathrm{y}$ : $\mathrm{x}$ is FTIR analysis, $\mathrm{y}$ is based on reconstruction of bulk inclusion composition by adding equilibrium gas (bubble) volume (see text).

${ }^{i}$ Saturation pressure calculated from $\mathrm{H}_{2} \mathrm{O}$ and $\mathrm{CO}_{2}$ concentrations of melt inclusions using VolatileCale program (Newman and Lowenstarn, 2002): $\mathrm{x} / \mathrm{y}$ : $\mathrm{x}$ is values for glass only: $\mathrm{y}$ is based on the corrected values in regard with bubble in inclusion. $\mathrm{If}^{\mathrm{CO}_{2}}$ concentration of the glass is below the detection limit. $\mathrm{y}$ is maximum estimate.

${ }^{\mathrm{j}}$ The inclusion contains an opaque mineral whose major component is iron oxide. Size of the mineral is $3 \mu \mathrm{m}$.

${ }^{\mathrm{k}}$ Groundmass of the bomb (819B1) whose whole-rock chemistry was determined (Table 1). Recalculated to $100 \%$ on a water free basis. Values in parenthesis represent the standard deviation of analyses.

${ }^{\mathrm{l}}$ Estimation based on back-scattered electron image of the inclusion. See text.

${ }^{\mathrm{m}}$ The low total value of this inclusion is probably due to charge up of electron beam. Analysis of its host phenocryst also gave low total, but similar Fo\% with the other type A olivine and correct value for ratio of cation to oxygen. As this suggests that ratios among major elements of the inclusion analysis are equal to the original values, we used the major element composition of the inclusion on discussion after it was recalculated to $100 \mathrm{wt} . \%$ on a water free basis. 